\title{
Research Progress on the Mechanism of College Students' Emotional Disorder and Sports Intervention Program
}

\author{
Li Zhang ${ }^{1}$, Xiaowan Song ${ }^{1}$, Hao Zhang ${ }^{2 *}$ \\ ${ }^{1}$ Department of Physical Education, China University of Mining and Technology, Beijing 100083, China \\ ${ }^{2}$ Department of Physical Education, Beijing University of Posts and Telecommunications, Beijing, 100876, \\ China \\ *Corresponding Author.
}

\begin{abstract}
:
At present, the problem of emotional disorders in college student has attracted more and more attention from the society, and mental health problems have become a common problem in the process of young people's growth, learning and life. Especially, the physiological characteristics of growth and development during university leads to the occurrence of anxiety, which require external intervention and guidance. Now, mental health education in China is generally absent, and the treatment of mental health is incomplete. There is a lack of scientific method guidance for mental health intervention and guidance for college students. The college students have just gone through the learning in universities. After entering the society, they are in a relatively relaxed state both in body and mind. This phase is also a stage when problems are easy to occur. This paper studies the relevant literature, analyzes the mechanism of college students' emotional disorder, and puts forward reasonable sports intervention programs.
\end{abstract}

Keywords: College student, Emotional disorders, Occurrence mechanism, Sports intervention

\section{INTRODUCTION}

In recent years, with the expansion of higher education, the reform and transformation of examination education and the change of population and family structure, different psychological stress factors have increased sharply. College students are in a period of rapid improvement from family life to independent social life, and the incidence of emotional disorders has risen sharply in the course of individual life. Faced with the pressure brought by problems such as study, friends, employment, regional and economic differences, it is easy for college students to have negative emotional and mental problems in their study and life. At 
present, emotional disorder has become the main psychological problem of college students, and most of them are negative emotions such as depression and anxiety [1].

According to the survey of professional institutions, the students who study and live in colleges and universities are more likely to suffer from depression, anxiety and other emotional disorders than the general youth. $40 \%$ of freshmen and over $50 \%$ of college graduates have psychological disorders at different degrees, which to some extent lead to serious physical and mental injuries such as daily study and life satisfaction of college students. Increasing psychological pressure has become one of the main reasons affecting physical and mental health and overall organizational efficiency of individuals [2]. Therefore, the role of emotional disorder mental health of college students plays a considerable role in the normal development of Chinese society.

At present, anxiety is the main emotional disorder of college students. According to the survey, emotional disorder is caused by the interaction of external factors such as family atmosphere and social environment, as well as the genetic, neurobiochemical and other factors of patients themselves [3]. At present, there is still a lack of early intervention research, especially the related research of sports intervention. Physical exercise, as a means of conditioning modern life style, can not only enhance the physical function of college students, but also have a close relationship with their psychological health and stability. Jiang Yuan and other studies have proved that sports activities can improve the emotional disorder of college students [4]. Therefore, the purpose of this study is to explore whether sports intervention can cure and solve college students' emotional disorder by analyzing various factors of college students' emotional disorder. At the same time, according to the connotation of anxiety, we can understand the occurrence mechanism of college students' emotional disorder caused by different influencing factors, use sports intervention means, carry out targeted intervention and health management of college students' emotional disorder, explore the occurrence mechanism of emotional disorder, build a model suitable for emotional disorder of college students in China, and finally put forward effective sports intervention programs.

\section{THE CONNOTATION OF ANXIETY}

Emotion is a kind of inner experience, and Beck [5] put forward the cognitive theory of emotional disorder. The international understanding of anxiety disorder is relatively unified. It is generally believed that emotional disorder is a group of diseases with anxiety, terror and depression as the main clinical manifestations [6], and the phenomenon of depression and anxiety accounts for $75 \%$ of patients with emotional disorder. From different angles, we can give multiple definitions of emotional disorder. Although there are some research deficiencies 
in each stage, these studies promote the research and development of emotional disorder theory [7].

Anxiety is a state of fear that human body can feel subjectively [8]. A negative emotional state like this generally thinks that noting can be done for difficulties or threats. Especially when an individual encounters an impact on his self-cognitive value, self-esteem and self-confidence, the more serious anxiety may develop into the so-called anxiety disorder. However, in daily life and study, if students have appropriate anxiety in the process of studying in physical education class, it will play a positive role. Therefore, anxiety is not always outdated and pathological. As the consciousness of existence and individual, it can regulate and even control the daily physiological activities of human body. Proper anxiety can actively mobilize the functions of various physiological organs of human body to a certain extent, and improve the stress response speed and acuity of brain. However, when some students' anxiety has a certain degree of pathological characteristics, it will form obstacles to their daily social and social functions, which will become morbid of emotional disorders. Anxiety is often associated with mental blows and threats that the human body cannot predict. Subjectively, anxiety, tension, discomfort, and even grief are accompanied by dysfunction of nervous system [9]. Therefore, PE teachers should pay enough attention to the positive effects brought by anxiety, and avoid and put an end to its negative effects as far as possible.

\section{THE OCCURRENCE MECHANISM OF EMOTIONAL DISORDERS}

Emotional disorders are mostly related to social and psychological factors [8]. Emotional anxiety is the main factor of college students' emotional disorder. Through the specific investigation and statistics of the influencing factors of college students' anxiety, these influencing factors mainly include physiological and social psychological factors. The survey found that parents' expectations and demands on their children will further enhance the incidence of emotional disorders. Contemporary college students tend to be influenced by the surrounding environment because of their unstable personality, showing great emotional fluctuations. At the same time, it is found that the gender difference of physiological changes during puberty leads to a higher incidence of emotional disorders among adolescent girls than boys [9]. The mechanism of emotional disorder is due to the problems in processing information in the central nervous system, which can be divided into two theoretical viewpoints: primary endogenous mechanism and reaction mechanism. The endogenous mechanism is emotional disorder caused by the dysfunction of neurotransmitters in the brain, especially serotonin $[10,11]$. The reaction mechanism refers to psychological problems such as anxiety, depression, loneliness and despair caused by psychological imbalance and cognitive misinterpretation due to various factors such as physiology, social environment and personality 
traits. They affect the effective ability of emotional management and regulation [12].

There are many reasons for anxiety, including situational anxiety, nervous anxiety and emotional anxiety. Situational anxiety often refers to anxiety caused by external psychological pressure, such as facing exams, studying or speaking in public. Emotional anxiety refers to anxiety caused by an individual's worry about what is expected to happen, or feeling remorse for his own fault or mistake. Nervous anxiety refers to anxiety caused by psychological and physiological reasons such as panic, insomnia, palpitation and emotional disorder. Anxiety disorders are a kind of mental disorder, and acute anxiety disorder is one of them. In addition, there is a kind of chronic anxiety disorder. The symptoms of the chronic anxiety disorder are different from those of acute anxiety disorder. The chronic anxiety disorder is accompanied by muscle tension, restlessness, uncontrolled limbs, sweating and other symptoms. Although patients with this emotional disorder know that they are already stressed out, they can't control themselves [13].

At present, some research achievements have been made in the study of emotional disorders, and preliminary consensus have been reached on the basic concepts and core concepts, which has laid a solid foundation for the follow-up research. Defining the connotation of emotional disorder and analyzing its influencing factors is of great significance to correctly understand the essence and influence of emotional order; aiming at college students, who are prone to emotional disorders, the internal root of their problems has not been studied in depth. However, there are no systematic and comprehensive methods, and there is still a lack of active research on emotional management of college students; in the measurement of emotional disorder, there are no uniform standards in China, and international measurement tables are mainly used for reference. Based on the existing scales[14], this paper analyzes the relevant influencing factors by studying domestic and foreign literature, including individual subjective evaluation and future expectation, and compiles the emotional disorder assessment scale suitable for college students by analyzing the variables of physiology, psychology and society, which reveals the current situation of college students' emotional disorder and provides theoretical guidance for analyzing the actual effect of physical exercise on college students' emotional disorder.

\section{RESEARCH PROGRESS ON AND MODEL CONSTRUCTION OF SPORTS INTERVENTION PROGRAM}

\subsection{Research on Sports Intervention}

The foothold of sports intervention program research lies in the intervention and treatment of college students' anxiety. Positive psychotherapy, which aims to cultivate positive emotion, 
behavior and cognition, can significantly improve happiness [15]. In China, the psychological counseling research of many active groups shows that active psychological group therapy can effectively alleviate the anxiety and depression of college students, and can effectively reduce the suicide rate of college students and improve their physical and mental health [16].

Studies have shown that better sports programs are effective in preventing adolescent depression. Research on the influence of physical exercise on psychological stress mainly focuses on lymphocytes, neutrophils, immune molecules, immunosuppression, etc. Physical exercise can not only improve immunity through body activities, but also improve immunity to viruses through affecting psychological factors such as emotions. Studies at home and abroad have found that physical exercise can effectively restrain negative psychological factors and form a good psychological state. Physical exercise can improve body immunity by antagonizing psychological stress and improving physical and mental state. Physical exercise can promote the brain to derive BDNF (Brain Derived Neurotrophic Factor), which helps the human body to produce positive mental states such as happiness and satisfaction.

The positive influence of daily physical exercise on college students' mental health cannot be replaced by any other means and methods of internal intervention. Some scholars have found that physical exercise, as a positive and effective intervention method, can not only improve students' self-esteem, but also play a positive role in promoting students' mental health. Physical self-esteem, also known as physical self-perception, refers to the emotional evaluation of an individual's physical intention or self-evaluation of his own physical characteristics and appearance [17, 18]. Physical exercise has a direct effect on individual self-efficacy. That is, the greater the intensity of physical exercise is, the higher the self-efficacy is. Physical exercise can improve teenagers' self-efficacy in two ways. The first is the direct effect. In the normal physical exercise process, one should be brave in facing adversity and difficulties. When one is recognized by the people around him, his sense of accomplishment will be improved and his sense of self-efficacy will also be improved. The second is the indirect effect. The improvement of self-efficacy can be indirectly improved by the improvement of self-esteem. After regular physical exercise, young people's self-esteem can be improved, and at the same time, their self-esteem can be indirectly improved [19]. It can be concluded from many studies that sports intervention can have a significant effect on human emotion regulation, and the emotion regulation ability of people taking regular physical exercise is stronger than that of low exercise frequency [20].

Many studies have found that long-lasting aerobic sports can play a positive role in relieving depression and anxiety. And its emotional regulation on human body is mainly reflected in the positive correlation between sports and individual psychological 
self-satisfaction. That is, individuals can absorb the corresponding positive emotions related to physical exercise in the process of participating in sports [17]. Participation in sports has a profound impact on human psychology, which has been unanimously recognized and proved by experts at home and abroad. The reason why sports can adjust people's emotional state is that those who participate in physical exercise can experience the corresponding happiness brought by sports. Some scholars believe that proper participation in sports can promote human body to release a substance called endorphin, which can make us feel happy. Therefore, taking physical exercise, especially favorable and familiar physical exercise, can make people have fun.

\subsection{Model Construction-sports Intervention Model for College Students' Emotional} Disorder

Through literature review and analysis, EAI is selected as the theoretical framework of "College Students' Sports Dependence Scale", and the current situation of college students' group physical exercise is interpreted from the perspectives of sports consciousness, sports motivation and sports behavior. The scale framework is formed through large sample investigation and screening items, and the applicability evaluation scale of sports ability of college students in China is formed; Delphi method is used for expert inquiry. The parameters of each dimension and the weight of each index of the ability assessment scale are defined, and the "College Students' Sports Dependence Scale" is established. Comprehensive analytic hierarchy process (AHP) is used to divide the ability grades to form the evaluation standard of college students' sports ability grades. At the same time, guided by the theory of health management and combined with the actual situation of college students, the College Students' Emotional Disorder Questionnaire is constructed to understand the emotional status and illness of college students, and provide information basis for later intervention [17].

The sports intervention has different effects on people with different degrees of psychological disorders. A detailed and comprehensive assessment of college students is made before formulating intervention programs. Information about students' psychology, abilities, preferences and individual actual need is collected, which helps researchers to make appropriate intervention plans and goals before intervention. In the process of physical exercise, different, representative and easy-to-implement physical exercise methods, such as strength, aerobic endurance and strength mixed exercises, are used to carry out experimental research on physical intervention for students with emotional disorders, so as to prove the influence of physical exercise on emotional disorders. At the same time, based on the proof of many experiments, it is concluded that different exercise methods and intensities have different effects on emotional disorders of students of different genders and related physiological factors, so as to provide more support for the theoretical research on the psychological benefits of 
physical exercise[18].

Through the previous structural equation, this paper analyzes the psychosocial variables that actually affect college students' emotional disorder at different time nodes (one year, two years, three years and above after illness). According to the main influencing factors of emotional disorder and under the theoretical guidance of "cognitive behavior theory" and "health belief model", a sports intervention time matching model (TSI) with "physiological intervention, psychological intervention and social intervention" as the core is established, namely implementing matching intervention based on influencing factors at different time points. That is to say, in the first year (P1) after the occurrence of emotional disorder, physiological intervention has a positive effect on the emotional disorder of college students, which can promote the development of nervous system and enhance the adaptability of the body; in the second year after the occurrence of emotional disorder (P2), psychological intervention is the core, which has a positive effect on the group emotional disorder of college students. Psychological intervention can alleviate psychological anxiety and tension, and help the human body release stress; in the third year (P3) after the occurrence of emotional disorder, social intervention, as the core, can play a positive role in the group emotional disorder of college students, and can alleviate the emotional problems of patients. At the same time, the sports intervention model of integrating health education organically at each time point to play a synergistic role is shown in Fig 1. 


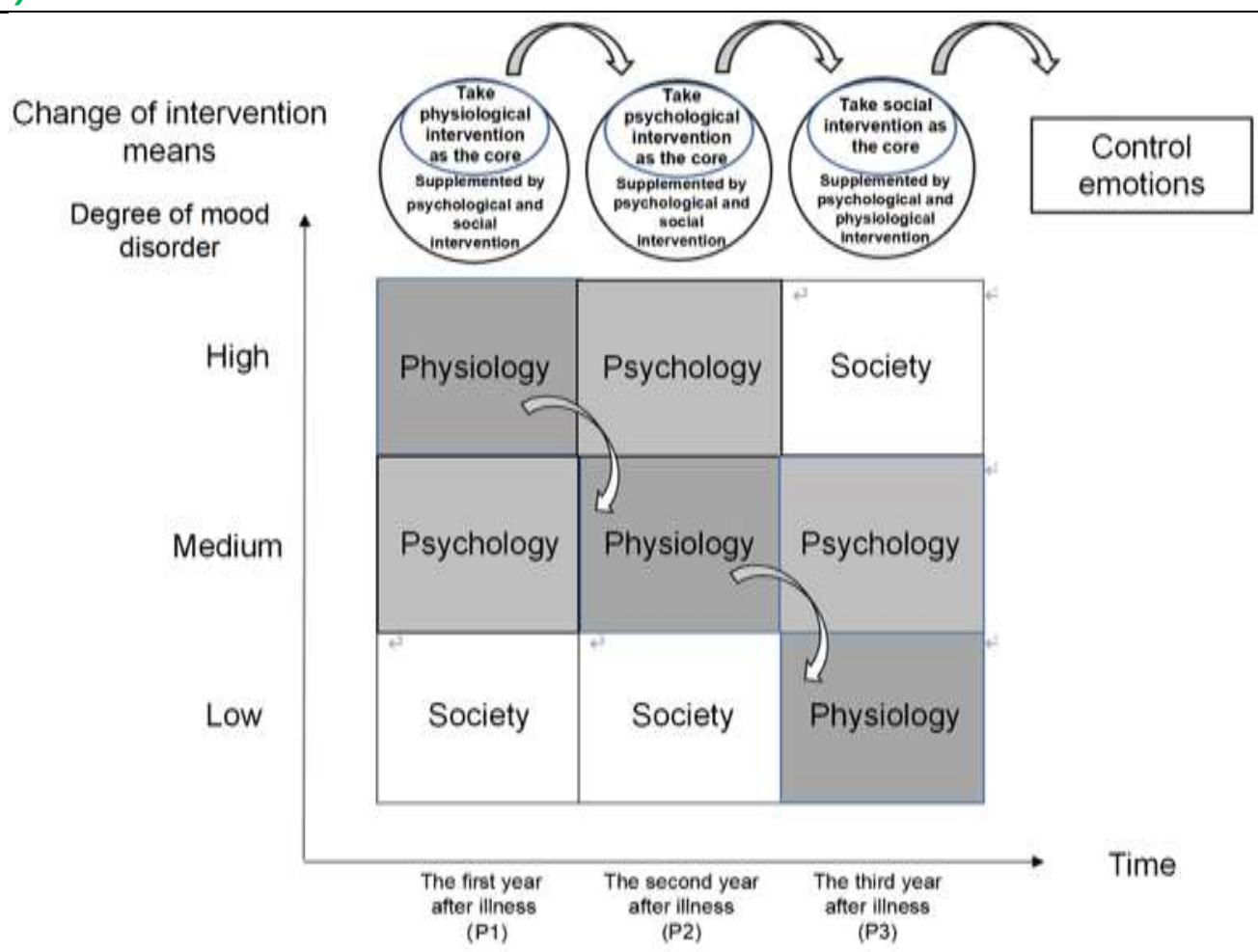

Fig 1: schematic diagram of stage matching of sports intervention model

\subsection{The Specific Implementation of the Research Process}

\subsubsection{Baseline survey}

College stage is a growing stage in which young people gradually grow mature. It plays a decisive role in the development of life. However, in this stage, new changes are taking place in terms of the physiology, emotion and surrounding environment of the youth group, which is a high-risk group with negative emotions, facing the pressure of heavy learning tasks, role conflicts, confused career development, and human-computer relationship troubles [19, 20]. In this study, different types of youth groups in colleges and universities from different regions are selected as research objects. Young people aged 17-23 and voluntarily participating in the test are recruited from colleges and universities. They are diagnosed with emotional disorders meeting the diagnostic criteria of emotional disorders in the third edition of Chinese Classification and Diagnostic Criteria for Mental Disorders (CCMD-3) by psychological counseling centers. At the same time90 cases including those diagnosed normal according to symptom checklist 90 (SCL-90) and those who have mental illness or serious physical illness are excluded from the study. Considering that a few patients with mild emotional disorder have 
no obvious symptoms and have not signed informed consent, and some severe patients have failed to join the group, selection bias may be caused [21, 22].

To ensure the reliability and validity of the results of large-scale questionnaire survey, this study optimizes the scale based on the results of small-scale questionnaire survey. First of all, relying on professional research websites, one hundred questionnaires are randomly distributed. Then, the exploratory factor analysis is carried out on the sports intervention variables, physiological guidance variables, psychological cognitive variables, social support variables and adjustment variables of the two questionnaires, and the indicators with low factor load value and unclear item attribution are eliminated according to the reliability value or factor analysis results [23].

4.3.2 Construction of time-matching intervention and health management model for patients with emotional disorders

Meta-analysis is used to systematically evaluate the related literature about emotional disorders at home and abroad, and the hypothetical model diagram of the occurrence process of emotional disorders is obtained (see Fig 2). According to the differences of influencing factors of emotional disorders at different time points, the school resources are effectively used to create a "three-aid" health management platform, and effective intervention means are found, refined and integrated. Taking "cognitive behavior theory" and "health belief model" as the theoretical basis for the design of experience intervention scheme in this study, "physiological intervention and psychological intervention" are established respectively [24].

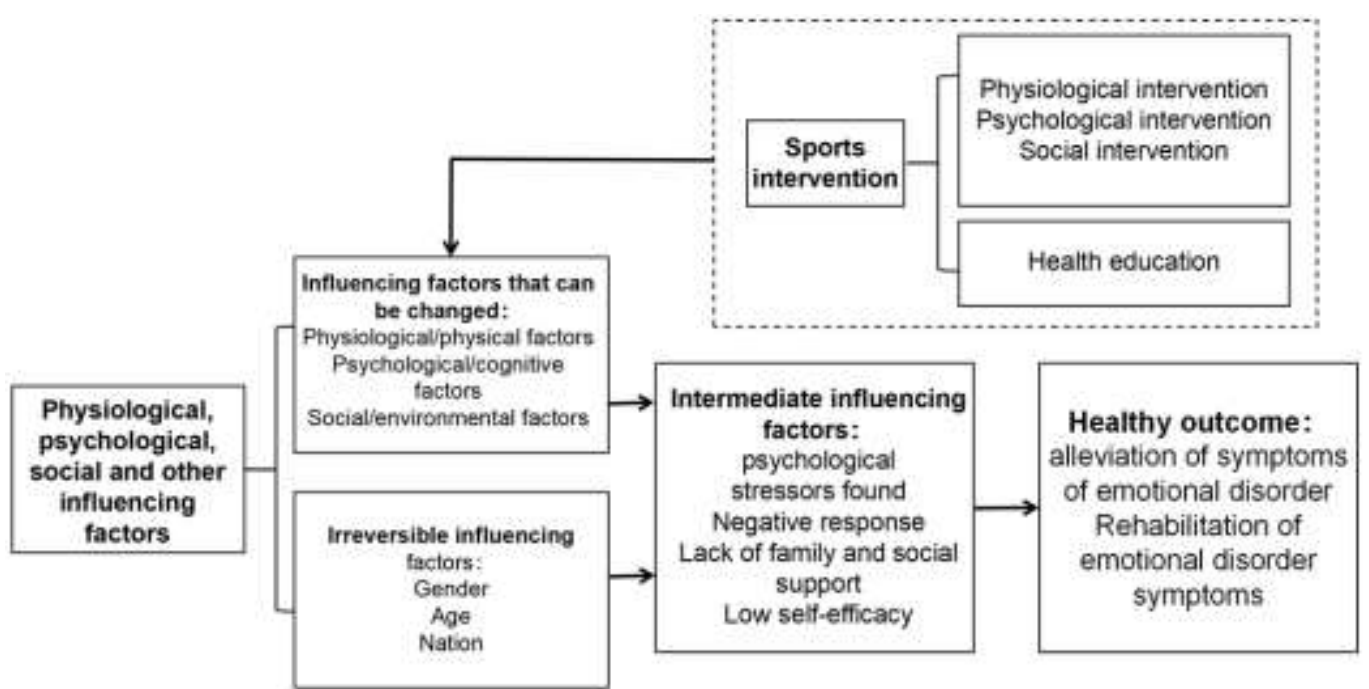

Fig 2: hypothetical model diagram of the occurrence process of emotional disorder 
According to the sports intervention model of college students' emotional disorder, school resources are effectively used to create a "three-aid" health management platform, that is, the health management platform of "school funding-family mutual assistance-patient self-help" (see Fig 3). Taking the prevention of college students' emotional disorder as the initial goal and "reducing the prevalence rate, improving the level of psychological adaptation and enhancing social function" as the management goal, and relying on the "three-aid" health management platform for emotional disorder, a health management model with TSI model as the core is constructed. Psychiatrists, psychological counseling and therapists are invited for the evaluation of the scientificity, applicability and feasibility [25-27].

The model of time-matching intervention and health management for emotional disorders is based on the "three-aid" (school funding-family mutual assistance-patient self-help) health management platform, with the time-matching intervention for emotional disorders of young people as the core. Chiesa et al. found that compared with the psychological education group with similar design and structure except the main MBCT components such as mindfulness meditation, the quality of life of the patients with mental disorder who were not relieved after 8 weeks of drug treatment was significantly improved after 26 weeks of MBCT treatment. Besides, the longer the treatment time is, the more obvious the effect is [28]. 


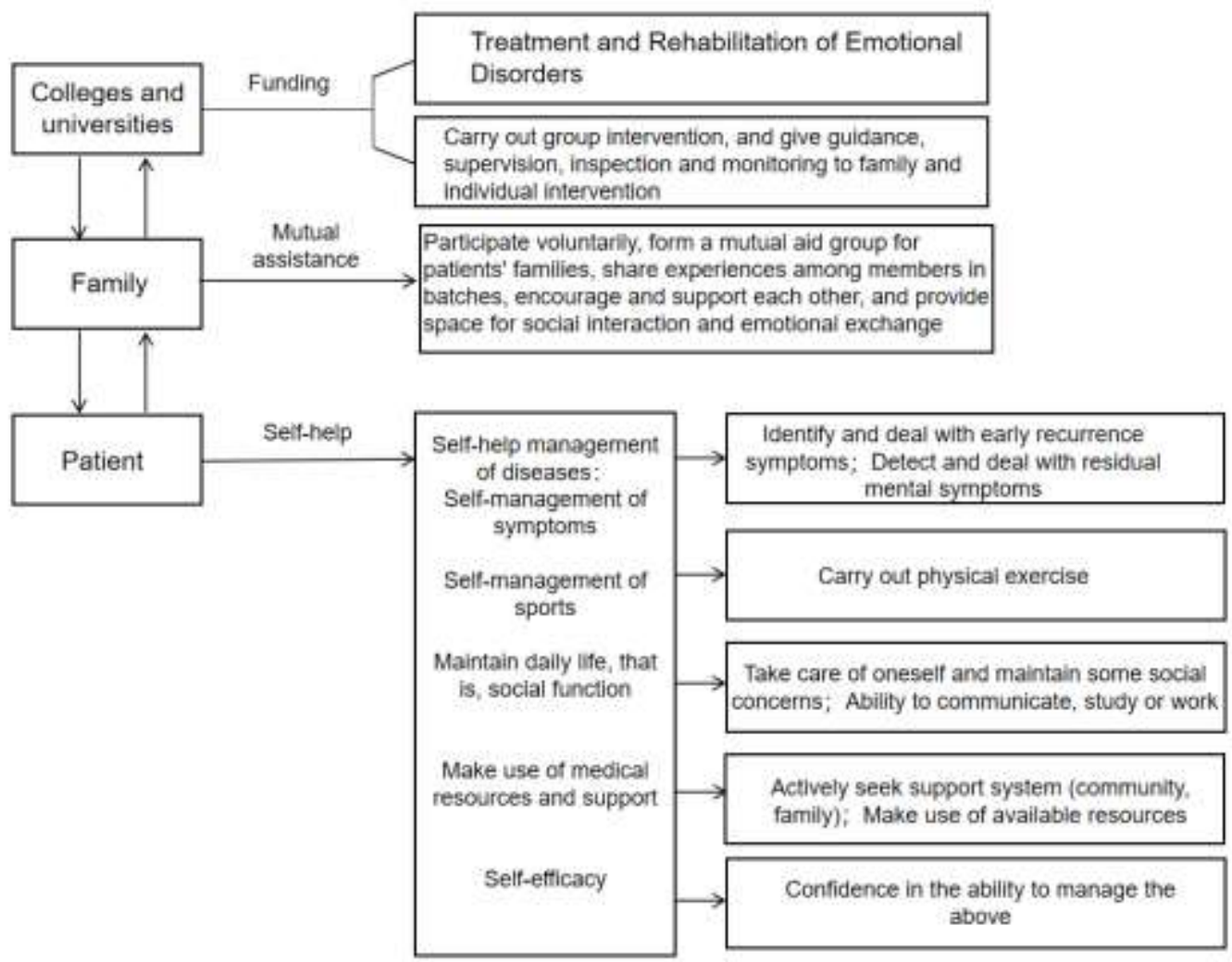

Fig 3: "three-aid" health management platform for emotional disorders

\section{CONCLUSION}

To sum up, sports intervention can improve college students' ability to resist setbacks and adjust their emotions, cultivate their strong will, innovative spirit and innovative ability, help them form a positive and optimistic attitude towards life, and effectively relieve their anxiety. Emotional disorder intervention is a systematic project from individual, school to society. This paper analyzes its performance, causes and influencing factors, establishes a sports intervention mechanism for college students' emotional disorder, and puts forward the logical structure of sports intervention mechanism and effective intervention measures. The purpose is to help schools establish and improve the intervention system for emotional disorders, improve the ability for the intervention of college students' emotional disorders, and improve college students' mental health.

\section{ACKNOWLEDGEMENTS}

This work was supported by the Fundamental Research Funds for the Central Universities 


\section{REFERENCES}

[1] Jin HY, Wang SH, Zhao Y (2011) Experimental study on metacognitive psychological intervention effect of college students with generalized anxiety disorder. China Journal of Health Psychology 19(07): 791-794.

[2] Wang XY, Lin S, Chen LY (2013) Research on the influence mechanism of research pressure on research performance-the moderating effect of academic atmosphere and emotional intelligence. Studies in Science of Science 31(10): 1564-1571.

[3] Xu H, Zhang WD (2004) Analysis of adolescent depression. Social Psychological Science 3: 106-109.

[4] Jiang Y, Zhang LW, Mao ZX (2015) Influencing factors of emotional benefits of physical exercise. Studies of Psychology and Behavior 13(03): 328-333.

[7] Wang ZH, Lv W, Du J, Wang KJ (2011) The relationship between positive emotions and mental health of college students: the mediating effect of personal resources. Chinese Mental Health Journal (07): 521-527.

[8] Zhang YJ, Kang HY, Chen J, Zhou LG (2009) The relationship between the behavior of children with emotional disorders and the way parents educate their children. China Journal of Health Psychology 17(02): 164-166.

[9] Li Y, Yu Q, Ma L, et al. (2008) Revalence of depression and anxiety symptoms and their influence factors during menopausal transition and postmenopause in Beijing city. Maturitas 61(3): 238-242.

[10] Mingione CJ, Heffner JL, Blom TJ, Anthenelli RM (2001) Childhood adversity, serotonin transporter(5-HTTLPR) genotype, and risk for cigarette smoking And nicotine dependence in alcohol dependent adults. Drug and Alcohol Dependence 1231-3.

[11] Wu S, An SC, Chen HB, Li F (2014) Regulation of dopamine D1 receptor on glutamate and its NMDA receptor in rat orbital frontal lobe during chronic stress depression. Acta Psychologica Sinica 46(01): 69-78.

[12] Xia G, Qian M (2001) The relationship of parenting style to self-report mental Health among two sub cultures of Chinese. Journal of Adolescent 24(2): 251-260.

[13] Dwairy M, Menshar KE, (2006) Parenting style, individuation, and mental health of Egypti An adolescents. Journal of Adolescence 29(1): 103-117.

[14] Chen XL (2020) Analysis of college students' anxiety and its regulation. The Farmers Consultant (11): 287.

[15] Xiao LQ (2007) The relationship between sports and college students' social adaptability. Journal of Physical Education 02: 79-82.

[16] Wang LP, Wang HP (2019) New progress in three psychotherapy studies on adolescent depression. Medicine \& Philosophy 40(03): 51-54.

[17] Diener (1984) Subject well-being. Psychological Bulletin (95): 542-575.

[18] Marie C, Richard P, Kate E, Katie D (2020) Address cognitive impairment in mood disorders: A role for the mental health nurse. Journal of Psychiatric and Mental Health Nursing 27(4). 
[19] Zhu XH (2020) 43 cases of adolescent emotional disorders and psychological intervention. Psychological Monthly 15(08): 28-2.

[20] Zhang XY, Zhang LY (2020) Research progress on intervention of emotional release therapy. Modern Clinical Nursing 19(04): 70-7.

[21] Liang XW, Xie MH (2020) Research on Crisis Intervention Strategies of Common Emotional Disorders of Undergraduate Students. Psychological Monthly 15 (01): 48.

[22] Sun XJ (2019) Psychological intervention and treatment analysis of adolescent emotional disorder. Electronic Journal of Clinical Medical Literature 6(42): 83-84.

[23] Zhang H, Ping L, Yan JW, Chen CF (2005) Physical exercise of the elderly and its influence on depressive disorder. Chinese Journal of Rehabilitation Theory and Practice (10):850-852.

[24] Reschly DJ (2000) The present and future status of school psychology in the United States. School Psychology Review 29(4): 507.

[25] Chen HY (2019) Research on Crisis Intervention Strategies of College Students' Common Emotional Disorders. Comparative Study of Cultural Innovation 3(15): 175-176.

[26] Li SL, Tao R, Zheng J (2018) Abnormal future scenario thinking of patients with emotional disorders and related interventions. Chinese Journal of Clinical Psychology 26(03): 506-511.

[27] Zhang WY, Liu GF, Wu YH (2012) Regression analysis of related factors between college students' mental health and physical exercise. Modern Preventive Medicine (05): 1162-1165.

[28] Gao YH, Wang M (1999) Family factors affecting mental health of rural high school students. Chinese Mental Health Journal. 